\title{
Vertebral Artery Hypoplasia in a Black Kenyan Population
}

\author{
Julius Ogeng'o, Beda Olabu, Rankeet Sinkeet, Nafula M. Ogeng'o, and Hemedi Elbusaid \\ Department of Human Anatomy, University of Nairobi, P.O. Box 30197, Nairobi 00100, Kenya \\ Correspondence should be addressed to Beda Olabu; otienobeda@gmail.com
}

Received 9 April 2014; Revised 30 June 2014; Accepted 30 June 2014; Published 16 September 2014

Academic Editor: Adriá Arboix Damut

Copyright (C) 2014 Julius Ogeng'o et al. This is an open access article distributed under the Creative Commons Attribution License, which permits unrestricted use, distribution, and reproduction in any medium, provided the original work is properly cited.

\begin{abstract}
This study examined the characteristics of vertebral artery hypoplasia in 346 arteries of adult black Kenyans. The circumference was measured on haematoxylin/eosin stained microscopic sections of the distal one-third of the intracranial vertebral arteries using scion image analyser. Internal diameter was calculated in millimetre. Data were analysed using SPSS version 16.0. Vertebral artery hypoplasia (diameter $<2.0 \mathrm{~mm}$ ) occurred in $100(28.9 \%)$ arteries. Sixty of these (17.3\%) were on the left and 40 (11.6\%) on the right. Sixty $(17.3 \%)$ were in females while $40(11.6 \%)$ were in males. The side and gender differences were statistically significant at confidence interval of $95 \%$. Frequency of vertebral artery hypoplasia was higher than in most other populations studied. The condition differs from that in other populations because it is more common on the left side and in females. We recommend ultrasound, angio-CT, or angio-MRI evaluation of vertebral arterial system before diagnostic or interventional procedures on posterior circulation.
\end{abstract}

\section{Introduction}

Vertebral artery hypoplasia (VAH) refers to those arteries with diameter of less than $2.0 \mathrm{~mm}$ [1-3]. This condition predisposes to posterior circulation stroke [4-7] and vertebral artery (VA) atherosclerosis $[2,8,9]$ and can be confused with pathological occlusion from, say, atherosclerosis or dissection [10]. It is also associated deformities of other arterial components of posterior circulation including basilar and posterior communicating arteries $[11,12]$. Characteristics of this condition are also important in selection and moulding of catheters during interventional neuroradiological procedures as well as mitigating complications of endovascular treatment and prognostication of cerebrovascular disease [11].

These characteristics of VAH show ethnic variation [13, 14]. As intracranial cerebral atherosclerosis becomes more common in Sub-Saharan African countries [15], there is need for data on African populations to inform management of disorders in posterior circulation. There are, however, currently few data from black African populations. This study, therefore, investigated the pattern of vertebral artery hypoplasia in an adult black population.

\section{Materials and Methods}

Two hundred and seventeen formalin fixed brains from adult black Kenyans obtained during autopsy at the Department of Human Anatomy, University of Nairobi, Kenya, were available for the study. Ethical approval was granted by the Kenyatta National Hospital/University of Nairobi Ethics and Research Committee. Forty-four were excluded-30 for suspected cerebrovascular disease and 14 for damage to the arteries. The cases of stroke and other cardiovascular disease were excluded to minimize the potential confounding effect of pathological conditions such as, for example, atherosclerotic, arterial narrowing. One hundred and seventy-three brains (99 males; 74 females, age range 20-79 years) were examined. Only cases of noncardiovascular death, as determined at autopsy, were included. The main causes of death were trauma (60.1\%), infections $(21.4 \%)$, malignancy (13.3\%), poisoning (3.5\%), and drowning (1.7\%). The age distribution of the cases is as shown in Table 1.

The cardiovascular risk factors revealed included alcohol $(32.3 \%)$, diabetes mellitus (23.4\%), cigarette smoking $(20.8 \%)$, and obesity (14.5\%). The brains were divided into 
TABLE 1: Age distribution of the population from which arteries were obtained.

\begin{tabular}{lccc}
\hline \multirow{2}{*}{ Age range (years) } & \multicolumn{2}{c}{ Frequency } & \multirow{2}{*}{ Total [\%] } \\
& Male & Female & \\
\hline $21-30$ & 15 & 11 & $26[15]$ \\
$31-40$ & 19 & 15 & $34[20]$ \\
$41-50$ & 26 & 19 & $45[26]$ \\
$51-60$ & 25 & 18 & $43[25]$ \\
$61-70$ & 10 & 9 & $19[11]$ \\
$71-80$ & 4 & 2 & $6[3]$ \\
\hline Total & 99 & 74 & $173[100]$ \\
\hline
\end{tabular}

those of males and females. Arachnoid mater was gently peeled from the brainstem to expose vertebral and basilar arteries. Two millimetre specimens taken from the distal one-third of the intracranial vertebral artery were fixed in $10 \%$ formalin and processed for paraffin embedding and sectioning. Ten five micron serial sections from each arterial site were stained with haematoxylin/eosin and examined with light microscope at magnification $\times 40$. The images taken by photomicroscope were digitized and internal circumference of each of the 10 sections from each site of the artery determined using scion ${ }^{\odot}$ image analyser. Only complete sections were included. Diameter in millimetres was calculated from the formula $D=C / \pi$ where $D$ is the diameter, $C$ is the circumference, and $\pi=3.14$. The average diameter of the 10 sections was taken to be the diameter of that artery. Vertebral artery hypoplasia was defined as internal diameter equal to or less than $2 \mathrm{~mm}$. Photographs of representative samples of asymmetry were taken using a high resolution digital camera. Data were analysed using SPSS version 16.0 for Windows. Gender differences were analysed using the Student's $t$-test at $95 \%$ confidence intervals where $P$ value $\leq 0.05$ was taken as significant. Results are presented in digital macrographs, tables, and bar charts.

\section{Results}

In all cases, two vertebral arteries joined to form the basilar artery. One hundred and two (29.5\%) were symmetrical (Figure 1(a)), while $234(70.5 \%)$ were asymmetrical, with right side dominance. The mean diameter for the right was $2.84 \mathrm{~mm}( \pm 0.43 \mathrm{~mm})$, while that for the left was 2.35 $( \pm 0.16 \mathrm{~mm})$. This bilateral asymmetry was statistically significant $(P=0.047)$.

The overall diameter ranged from $0.4 \mathrm{~mm}$ to $3.4 \mathrm{~mm}$ with a mean of $2.65( \pm 0.16 \mathrm{~mm})$. The mean diameter was higher in females $(3.13 \mathrm{~mm})$ than that in males $(2.29 \mathrm{~mm})$. The difference was statistically significant $(P=0.034)$. The peak diameter range was $2.6-3.0 \mathrm{~mm}$, present in $136(39.3 \%)$, followed by $2.1-2.5 \mathrm{~mm}(20.2 \%)$. Forty arteries (11.6\%) measured $>3 \mathrm{~mm}$. Two hundred and forty-six (71.1\%) were normal with diameter $>2.0 \mathrm{~mm}$. One hundred cases $(28.9 \%)$ measured $2.0 \mathrm{~mm}$ and less; that is, they were hypoplastic (Figure 2). Of these hypoplastic ones 48 (13.9\%) measured 1.6-2.0 mm; that is, they were mildly hypoplastic (Figures $1(\mathrm{~b})$ and $1(\mathrm{c})$ ).
TABLE 2: Gender distribution of VAH among adult black Kenyans.

\begin{tabular}{lcc}
\hline Range & Male & Distribution \\
& 6 & Female \\
\hline $0-0.5$ & 10 & 4 \\
$0.6-1.0$ & 16 & 6 \\
$1.1-1.5$ & 28 & 8 \\
$1.6-2.0$ & 60 & 22 \\
\hline Total & & 40 \\
\hline
\end{tabular}

Twenty-six (7.5\%) had a diameter of less than $1.0 \mathrm{~mm}$ and may be considered severely hypoplastic (Figure 1(d)). Twentyeight of the hypoplastic arteries were left compared to 20 on the right. Sixty (17.3\%) of the hypoplastic arteries were in males, while $40(11.6 \%)$ were in females.

Among those with VAH, 60 were in males and 40 in females. The mean diameter for females was $3.14 \mathrm{~mm}$, while that for males was $2.29 \mathrm{~mm}$. The difference was statistically significant $(P=0.034)$ (Table 2).

\section{Discussion}

Observations of the current study that only $29.5 \%$ of vertebral arteries are symmetrical are consistent with the literature reports [16-18]. This asymmetry reflected in volume of blood flow has been related to origin of left subclavian artery directly from the aorta and angle of origin of vertebral artery [18]. Mean diameter of the vertebral artery of $2.65 \mathrm{~mm}$ is comparable to $2.6 \mathrm{~mm}$ reported for a study on an English population [19] but higher than $1.73 \mathrm{~mm}$ reported for a South African population [20]. It is lower than 3.25 for an Iranian population [18], $3.15 \mathrm{~mm}$ in Indian population [17], and $3.08 \mathrm{~mm}$ in Turkish [21] population.

These figures indicate wide variations in the diameter of vertebral arteries. Although such variations may be attributed to methodological factors, they probably reflect population differences [13].

The current study reveals a $28.9 \%$ prevalence of VAH, higher than $2.34-26.5 \%$ reported for most Caucasian [22$25]$ and Indo-Asian [2, 26-28] populations. Individuals with VAH have a high probability of posterior circulation stroke, with atherosclerotic susceptibility, ipsilateral lesions in the vertebral artery territory [2], and migraine with aura [29]. Indeed, even asymptomatic subjects with VAH have a significantly lower net VA flow volume and higher frequency of VA flow insufficiency [30]. Further, VAH serves as an independent factor of reduction of the posterior circulation blood flow velocity. It can also play a negative role in cases of occlusion of a major blood vessel since it limits potential compensatory blood circulation. In this way, it may lead to regional hypo-perfusion and complex neurovascular consequences which correspond to vestibular neuritis and migraine [3]. Accordingly, nearly $30 \%$ of the Kenyan population may be at risk of posterior circulation stroke and the other complications. This implies that in patients who present with vertebrobasilar insufficiency and aural migraine VAH should be considered. 


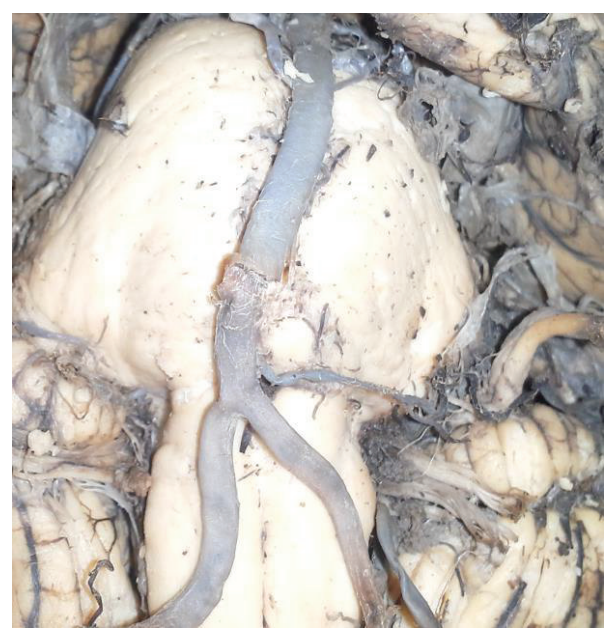

(a) Symmetrical vertebral arteries uniting to form basilar artery. Note the equal size of the vertebral arteries

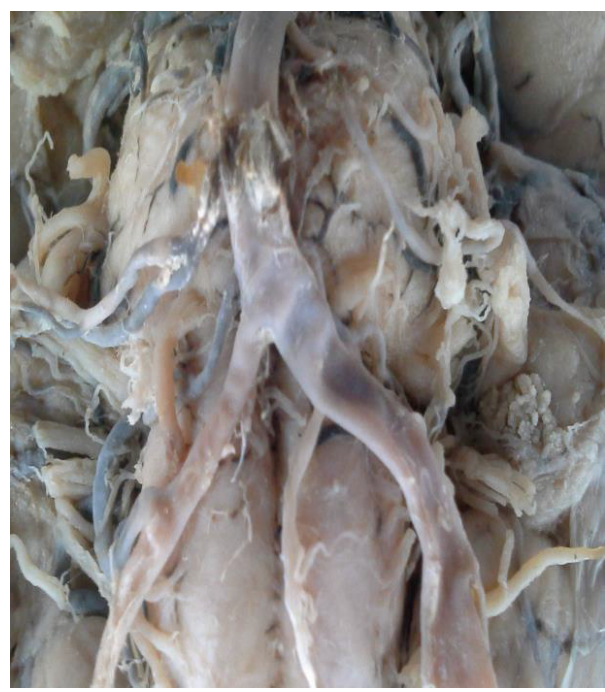

(c) Hypoplasia of RVA. Note that calibre RVA is less than half that of LVA

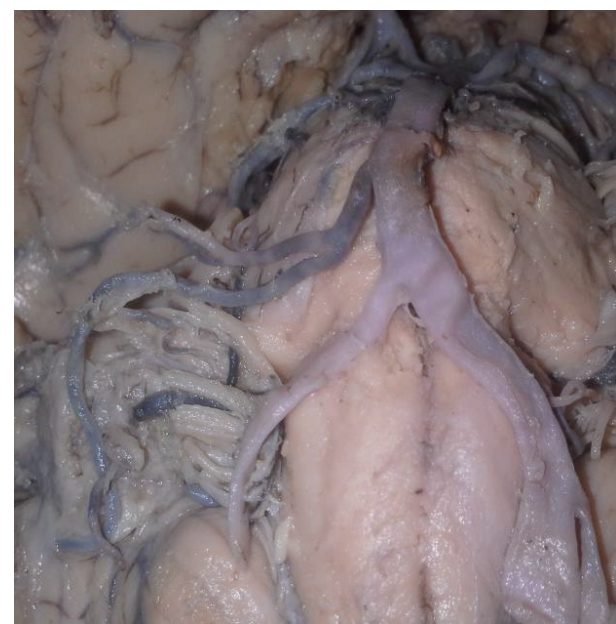

(b) Bilateral asymmetry with mild hypoplasia of right vertebral artery. Note the relatively smaller RVA compared to LVA

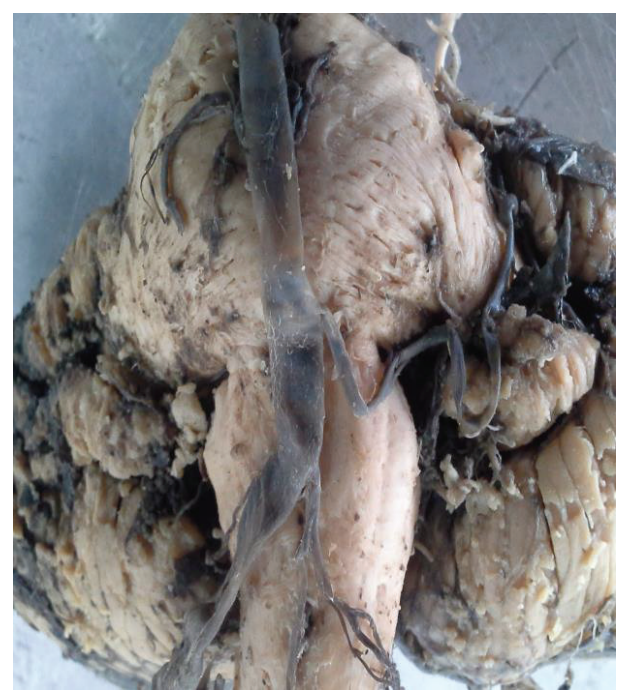

(d) Severe hypoplasia of the LVA. Note the obviously attenuated LVA relative to the normal RVA

FIGURE 1: (a)-(d) Photomicrographs showing various grades of vertebral artery hypoplasia in an adult black Kenyan population. BA: basilar artery; VA: vertebral artery; LVA: left vertebral artery; RBA: right vertebral artery.

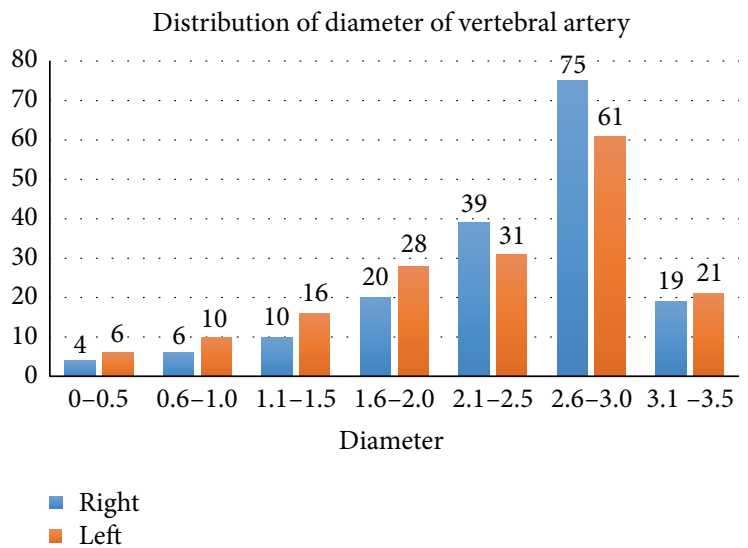

FIGURE 2: Distribution of diameter of vertebral artery in an adult black Kenyan population. 
The most remarkable observation of the present study is the left side predominance of VAH. This is at variance with most literature reports which show right side predominance $[8,9,22,28]$.

The present study reveals a male predominance, at variance with contemporary literature reports $[5,22,31]$. Although the studies may not be absolutely comparable because of differences in cutoff levels [3] and methodology, this diversity may reflect population variations probably based on epigenetic factors. Pertinent to this suggestions are assertions that the condition is hereditary [32, 33]. Accordingly, such population differences should be borne in mind during diagnostic and interventional neuroradiological and neurosurgical procedures on posterior circulation.

\section{Conclusion}

Vertebral artery hypoplasia occurs in about 30\% of black Kenyans studied, higher than in most other populations studied. It also differs from other populations in that it is more common on the left side and in females. We recommend ultrasound, CT-angio or angio-MRI evaluation of vertebral arterial system before diagnostic or interventional neurosurgical and neuroradiological procedures on posterior circulation.

\section{Conflict of Interests}

The authors declare that there is no conflict of interests regarding the publication of this paper.

\section{Acknowledgments}

The authors are grateful to Jacob Gimongo, Acleus Murunga, and Pamela Pande for Technical assistance and Antonina Odock-Opiko for typing the paper.

\section{References}

[1] C. M. Fisher, I. Gore, N. Okabe, and P. D. White, "Atherosclerosis of the carotid and vertebral arteries-extracranial and intracranial," Journal of Neuropathology \& Experimental Neurology, vol. 24, pp. 455-476, 1965.

[2] J. Park, J. Kim, and J. Roh, "Hypoplastic vertebral artery: frequency and associations with ischaemic stroke territory," Journal of Neurology, Neurosurgery and Psychiatry, vol. 78, no. 9, pp. 954-958, 2007.

[3] Y. Chuang, L. Chan, H. Wu, S. Lee, and Y. Chu, "The clinical relevance of vertebral artery hypoplasia," Acta Neurologica Taiwanica, vol. 21, no. 1, pp. 1-7, 2012.

[4] L. R. Caplan and R. Baker, "Extracranial occlusive vascular disease: does size matter?” Stroke, vol. 11, no. 1, pp. 63-66, 1980.

[5] J. Jeng and P. Yip, "Evaluation of vertebral artery hypoplasia and asymmetry by color-coded duplex ultrasonography," Ultrasound in Medicine and Biology, vol. 30, no. 5, pp. 605-609, 2004.

[6] A. S. Szárazová, E. Bartels, and P. Turčáni, "Vertebral artery hypoplasia and the posterior circulation stroke," Perspectives in Medicine, vol. 1, no. 1-12, pp. 198-202, 2012.
[7] X.-Y. Hu, Z.-H. Li, H.-Q. Liu et al., "Relationship between vertebral artery hypoplasia and posterior circulation stroke in Chinese patients," Neuroradiology, vol. 55, no. 3, pp. 291-295, 2013.

[8] A. H. Katsanos, M. Kosmidou, and S. Giannopoulos, "Vertebral artery hypoplasia in posterior circulation cerebral ischemia," Clinical Neurology and Neurosurgery, vol. 115, no. 7, pp. 11941195, 2013.

[9] A. H. Katsanos, M. Kosmidou, A. P. Kyritsis, and S. Giannopoulos, "Is vertebral artery hypoplasia a predisposing factor for posterior circulation cerebral ischaemia events? A comprenhensive review," European Neurology, vol. 70, no. 1-2, pp. 78-83, 2013.

[10] T. M. Buckenham and I. A. Wright, "Ultrasound of the extracranial vertebral artery," British Journal of Radiology, vol. 77, no. 913, pp. 15-20, 2004.

[11] S. Chaturvedi, T. G. Lukovits, W. Chen, and P. B. Gorelick, "Ischemia in the territory of a hypoplastic vertebrobasilar system," Neurology, vol. 52, no. 5, pp. 980-984, 1999.

[12] A. Vilmas, E. Barkauskas, A. Vilionskies, J. Rudzinskaite, and R. Moikunate, "Vertebral Artery Hypoplasia: importance for stroke development, the role of posterior communicating artery, possibility for surgical and conservative treatment," Acta Medica Lituanica, vol. 10, no. 2, pp. 110-114, 2003.

[13] G. C. Cloud and H. S. Markus, "Diagnosis and management of vertebral artery stenosis,” QJM, vol. 96, no. 1, pp. 27-34, 2003.

[14] J. G. Ciríaco, C. C. Leite, M. G. Martin et al., "Basilar artery occlusive disease in stroke survivors in a multiethnic population," Clinical Neurology and Neurosurgery, vol. 112, no. 3, pp. 233-236, 2010.

[15] O. O. Oladapo, J. Olusakiu, J. O. Ogun, and E. Akang, "Atherosclerosis of intracranial carotid arteries in Nigerians. A pilot autopsy study," Nigerian Journal of Cardiolog, vol. 10, pp. 62-67, 2013.

[16] M. Watanabe, A. Takahashi, Y. Hashizume, Y. Motegi, and K. Furuse, "The correlation between vertebral artery asymmetry and pontine infarction-an MR angiography study," Clinical Neurology, vol. 32, no. 7, pp. 708-712, 1992.

[17] B. S. Pai, R. G. Varma, R. N. Kulkarni, S. Nirmala, L. C. Manjunath, and S. Rakshith, "Microsurgical anatomy of the posterior circulation," Neurology India, vol. 55, no. 1, pp. 31-41, 2007.

[18] M. Nemati, A. S. Bavil, and N. Taheri, "Comparison of normal values of Duplex indices of vertebral arteries in young and elderly adults," Cardiovascular Ultrasound, vol. 7, no. 1, article 2, 2009.

[19] Y. L. Yu, I. F. Moseley, P. Pullicino, and W. I. McDonald, "The clinical picture of ectasia of the intracerebral arteries," Journal of Neurology, Neurosurgery and Psychiatry, vol. 45, no. 1, pp. 2936, 1982.

[20] J. Mitchell and A. McKay, "Comparison of left and right vertebral artery intracranial diameters," Anatomical Record, vol. 242, no. 3, pp. 350-354, 1995.

[21] V. Akgun, B. Battal, Y. Bozkurt et al., "Normal anatomical features and variations of the vertebrobasilar circulation and its branches: an analysis with 64-detector row CT and 3T MR angiographies," The Scientific World Journal, vol. 2013, Article ID 620162, 7 pages, 2013.

[22] S. Morović, T. Škarić-Jurić, and V. Demarin, "Vertebral artery hypoplasia: characteristics in a Croatian population sample," Acta Clinica Croatica, vol. 45, no. 4, pp. 325-329, 2006. 
[23] A. Songur, Y. Gonul, O. A. Ozen et al., "Variations in the intracranial vertebrobasilar system," Surgical and Radiologic Anatomy, vol. 30, no. 3, pp. 257-264, 2008.

[24] B. Oder, W. Oder, W. Lang, E. Marschnigg, and L. Deecke, "Hypoplasia, stenosis and other alterations of the vertebral artery: does impaired blood rheology manifest a hidden disease?” Acta Neurologica Scandinavica, vol. 97, no. 6, pp. 398403, 1998

[25] M. Sabau, A. Comanescu, and I. Popa, "Clinico-imagistical study of vertebral and basilar artery abnormalities in stroke patients," Proceedings of the 21st Meeting of European Neurological Society, 2011.

[26] J. Min and Y. Lee, "Transcranial Doppler ultrasonographic evaluation of vertebral artery hypoplasia and aplasia," Journal of the Neurological Sciences, vol. 260, no. 1-2, pp. 183-187, 2007.

[27] K. Morimoto, M. Nagahata, S. Ono et al., "Incidence of unilateral distal vertebral artery aplasia: evaluation by combining basiparallel anatomic scanning-magnetic resonance imaging (BPAS-MRI) and magnetic resonance angiography," Japanese Journal of Radiology, vol. 27, no. 3, pp. 151-155, 2009.

[28] Y. M. Chuang, C. C. Chen, and C. P. Lin, "Vertebral artery hypoplasia may contribute to abnormal vestibular evoked myogenic potentials," Acta Neurologica Taiwanica, vol. 18, no. 2, pp. 113-117, 2009.

[29] A. Lovrenčić-Huzjan, V. Demarin, T. Rundek, and V. Vuković, "Role of vertebral artery hypoplasia in migraine," Cephalalgia, vol. 18, no. 10, pp. 684-686, 1998.

[30] Y. Y. Chen, A. C. Chao, H. Y. Hsu, C. P. Chung, and H. H. Hu, "Vertebral artery hypoplasia is associated with a decrease in net vertebral flow volume," Ultrasound in Medicine and Biology, vol. 36, no. 1, pp. 38-43, 2010.

[31] C. Peterson, L. Phillips, A. Linden, and W. Hsu, "Vertebral artery hypoplasia: prevalence and reliability of identifying and grading its severity on magnetic resonance imaging scans," Journal of Manipulative and Physiological Therapeutics, vol. 33, no. 3, pp. 207-211, 2010.

[32] V. Demarin, T. Škarić-Jurić, A. Lovrenčić-Huzjan, M. Bosnar Puretić, and V. Vuković, "Vertebral artery hypoplasia: sex specific frequencies in 36 parent-offspring pairs," Collegium Antropologicum, vol. 25, no. 2, pp. 501-509, 2001.

[33] S. Iqbal, "A comprehensive study of the anatomical variations of the circle of Willis in adult Human Brains," Journal of Clinical and Diagnostic Research, vol. 7, no. 11, pp. 2423-2427, 2013. 

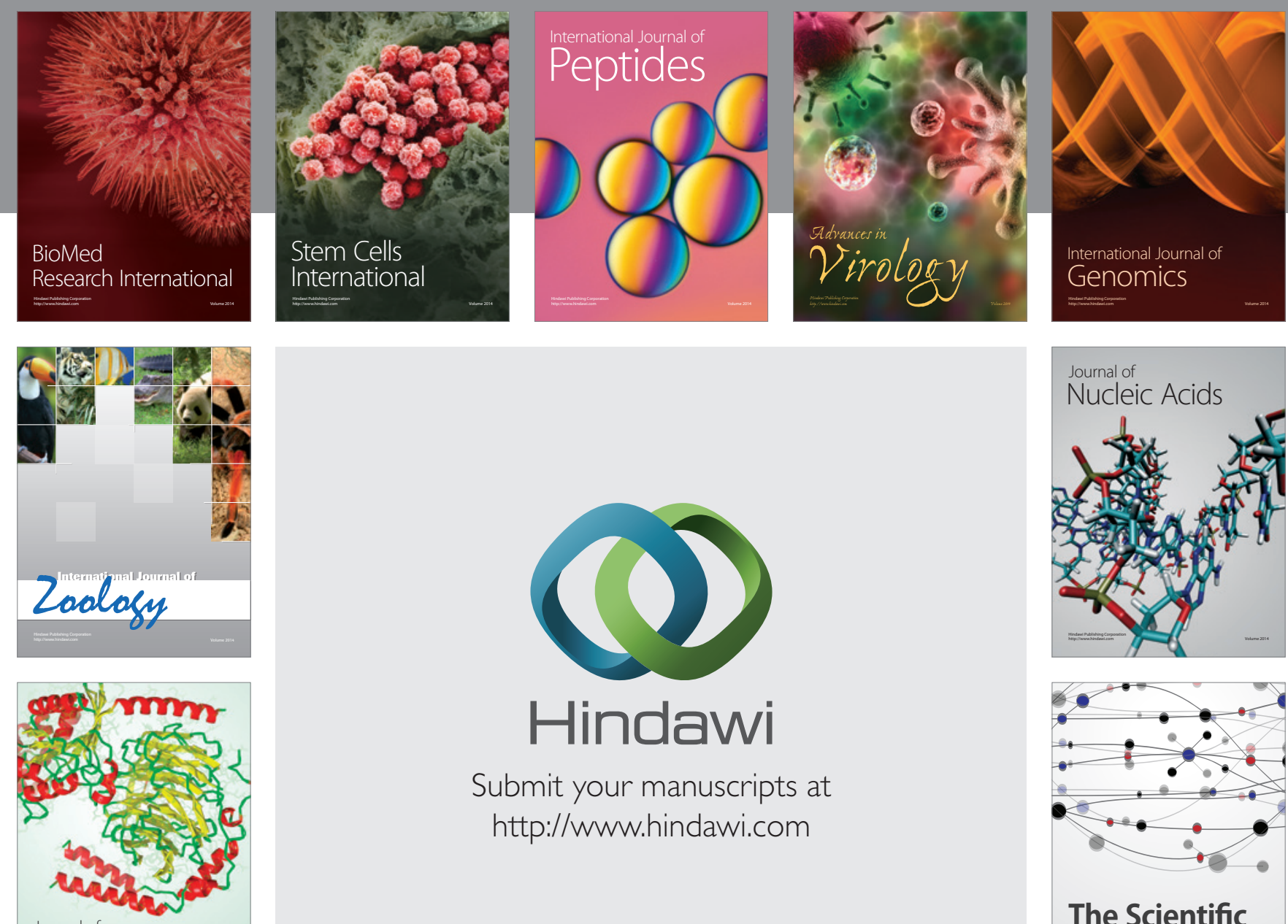

Submit your manuscripts at

http://www.hindawi.com

Journal of
Signal Transduction
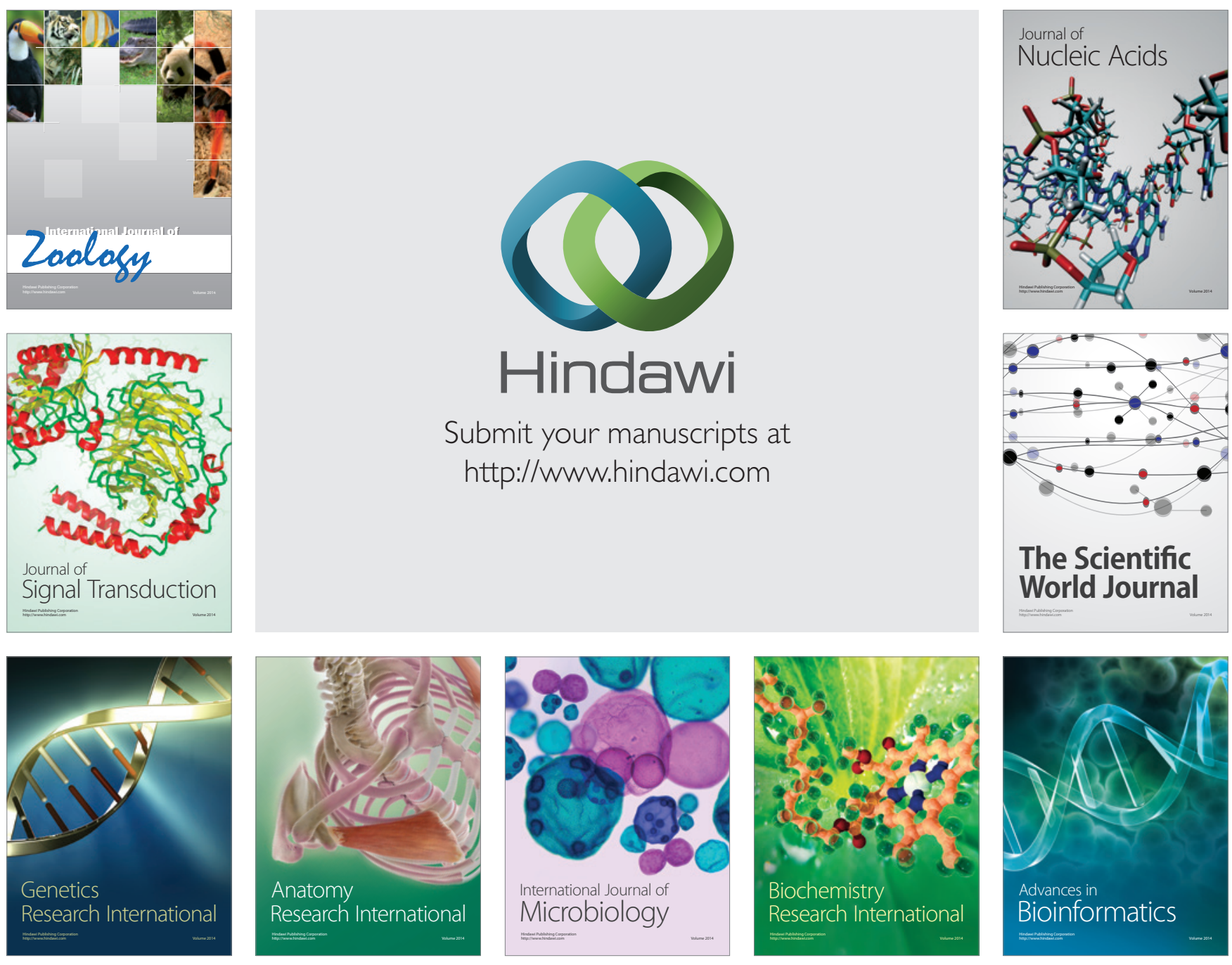

The Scientific World Journal
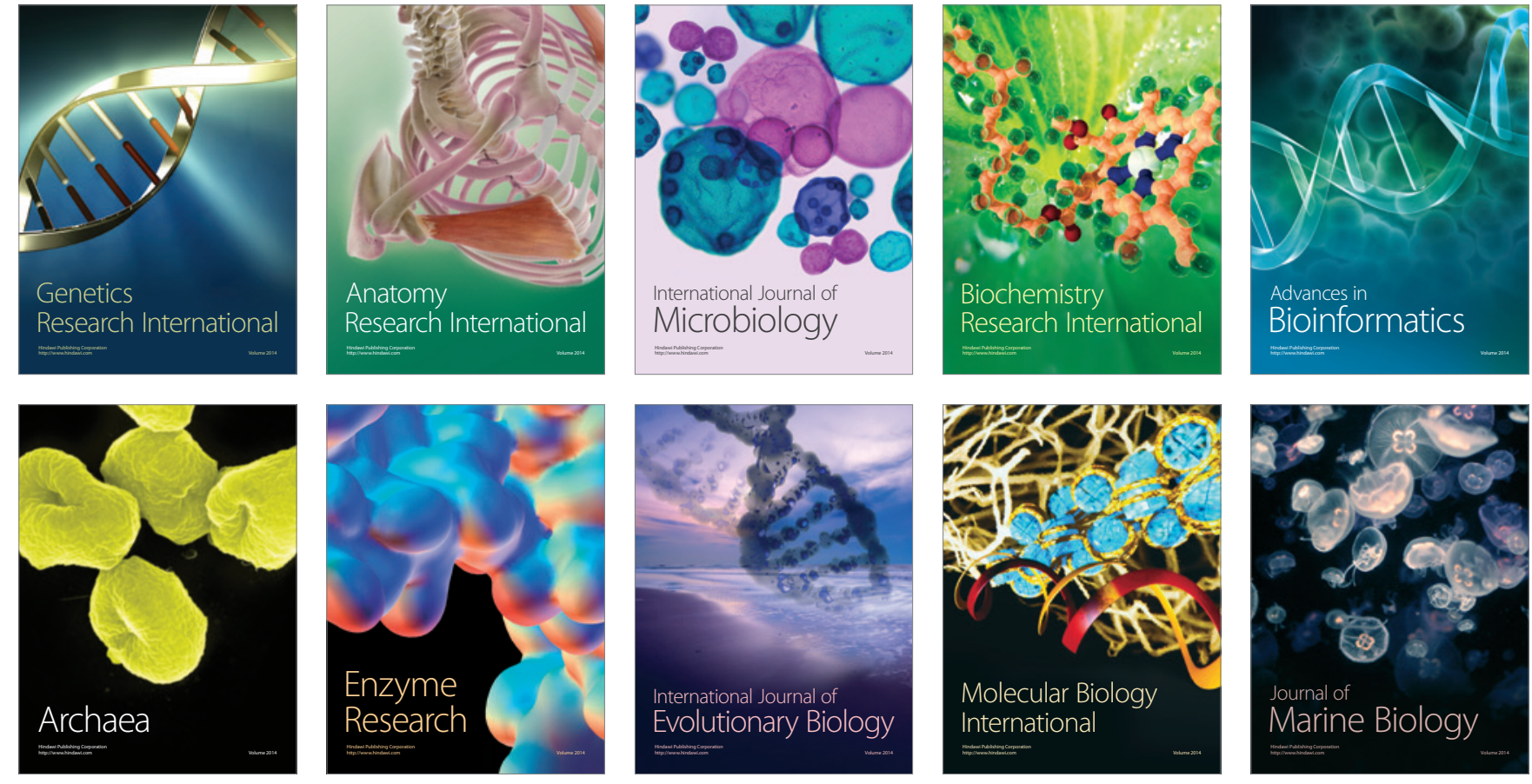